\title{
Perinevoid Alopecia: A Case Report
}

\author{
León Felipe Ruiz-Arriaga ${ }^{a}$ Lirio López-Garcíab María Elisa Vega-Memije ${ }^{a}$ \\ a Department of Dermatopathology, “Dr. Manuel Gea González” General Hospital, Mexico City, Mexico; \\ ${ }^{b}$ Dermatologist, Private Practice, Hospital Ángeles del Pedregal, Mexico City, Mexico
}

\section{Established Facts}

- Perinevoid alopecia is extremely rare.

- Only 5 cases have been reported in the literature.

\section{Novel Insights}

- Complete histopathologic description of the non-scarring alopecia compatible with alopecia areata.

\section{Keywords}

Perinevoid alopecia $\cdot$ Nevocentric alopecia $\cdot$ Halo nevus

\section{Abstract}

Perinevoid alopecia has been described as an associated alopecia surrounding a pigmented nevus due to an inflammatory response against nevus structures. It is described as a part of other nevocentric phenomena in which a cellular inflammatory response against nevus antigens develops. A 35-year-old male presented with a unique area of non-scarring alopecia surrounding an asymptomatic, pigmented nevus with a 1-month evolution. Trichoscopic and histologic findings were compatible with alopecia areata (AA). One month after excision, hair regrowth was reported by the patient. We concluded that perinevoid alopecia is an extremely rare clinical presentation of AA associated with a central, pigmented nevus where a cellular inflammatory response is triggered against hair follicles, nevoid cells, and melanocytic structures.

(c) 2018 S. Karger AG, Basel

\section{Introduction}

Pigmented nevi can present diverse inflammatory phenomena, the halo nevus being one of the most studied and prevalent ones. The halo nevus is a nevus with a hypopigmented peripheric area due to an autoimmune cellular response against nevus cell antigens [1]. Other nevocentric phenomena are perinevoid alopecia, erythema multiforme, halo eczema, and targetoid halo, among others.

Perinevoid alopecia, also known as perinevic alopecia or nevocentric alopecia, was first described by Yesudian and Thambiah [2] in a series of 3 cases. They associated alopecia with a central pigmented nevocellular nevus. It is thought that the alopecia is secondary to an inflammatory response against nevic cells or melanocytic structures.

The most representative histopathologic features of this condition are (1) the presence of nevus cells in nests surrounded by a dense, mononuclear infiltrate and lo-

\section{KARGER}

๑c 2018 S. Karger AG, Basel

E-Mail karger@karger.com

www.karger.com/sad
María Elisa Vega-Memije, MD, Department of Dermatopathology

"Dr. Manuel Gea González" General Hospital, Calz. de Tlalpan 4800, Belisario Domínguez Secc 16

Mexico City, 14080 (Mexico)

E-Mail elisavega50@gmail.com 

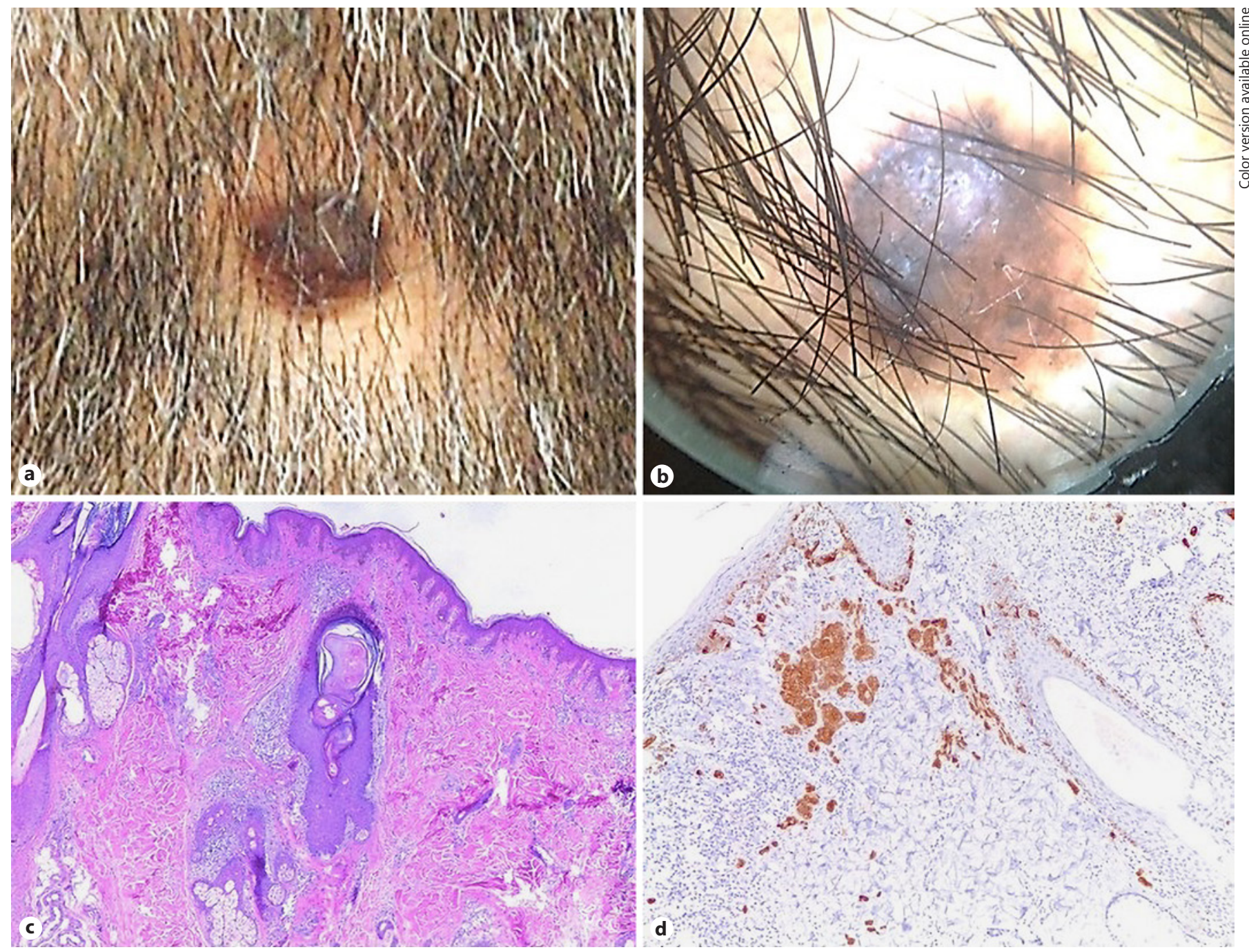

Fig. 1. a Macroscopic image of the alopecia patch surrounding a melanocytic nevus. b Dermoscopic image of the lesion showing an area of alopecia surrounding a melanocytic lesion, some exclamation mark hairs, and yellow dots. c Skin section stained with H\&E

showing the presence of an intradermal melanocytic nevus, hair follicles, and an intense lymphocytic infiltrate in the superficial and middle dermis surrounding hair follicles (magnification $\times 4$ ). d Melan-A stain positive in melanocyte nests (magnification $\times 10$ ).

cated around hair follicles, (2) fibrotic replacement of hair follicles, and (3) positive Alcian blue staining in the superficial dermis and around some hair follicles.

\section{Case Report}

We report the case of a 35-year-old male from Mexico City who presented a unique area of alopecia surrounding a pigmented nevus. He referred that the lesion had an evolution of 1 month and that it was asymptomatic. Clinically, it was a cupuliform, darkbrown pigmented tumor with peripheral areas of light-brown pigment, surrounded by an area of non-scarring alopecia with signs

of alopecia areata (AA; exclamation mark hairs, yellow dots, and vellus hairs) (Fig. 1).

An excisional biopsy was performed and sent to the dermatopathology department with the differential diagnosis of dysplastic nevi versus melanoma.

Histological findings were basket weave pattern stratum corneum with melanin pigment, and an epidermis with irregular acanthosis, proliferation of isolated melanocytes, and some of them forming nests in the basal layer. The cells were irregular, large, and hyperchromatic with loss of cohesion and with abundant melanin pigment in their interior, descending to the superficial dermis and gather in nests. In the dermis, cells tend to be smaller and regular, with an open chromatin pattern surrounded by a dense inflammatory infiltrate composed of lymphocytes. There is a deposit of me- 
lanic pigment and melanophages. Below the melanocytic tumor, there is a decrease in the number of hair follicles. The adjacent ones are miniaturized and conserve the sebaceous glands. There is an inflammatory infiltrate by lymphocytes in the upper third and in the bulb of some of the affected follicles; they are dilated and have mucin in their interior (Fig. 1c). There are also some fibrous stellae. The histological diagnosis of melanocytic compound nevi with inflammatory infiltrate and non-scarring alopecia was made.

One month after surgical excision, hair regrowth was observed. Neither recurrence of the lesion nor recurrence of the alopecia has been referred by the patient until now.

\section{Discussion}

Alopecias are divided in scarring and non-scarring alopecias for their study and clinical approach. AA is one of the non-scarring alopecias where an autoimmune response directed at the hair follicles is triggered that results in hair loss [3]. It is well described that in AA, the autoimmune process is mediated by T-lymphocytes, specially CD4+ and CD8+ T cells; however, the specific autoantigens are still being studied, and some hypotheses about the role of melanocytes' autoantigens in the pathogenesis of this process have been published $[4,5]$.

Perinevoid alopecia is considered a subset of AA because of the trichoscopic findings reported in isolated case reports such as exclamation mark hairs and black dots, and because of its spontaneous clinical history. We presented the case of a patient who noted the bald patch 1 month before consulting us. Except for the case reported by Mastruserio et al. [6], almost all cases have been reported around pigmented nevi, including our case [1, 7].
The most representative histopathologic features are (1) the presence of nevic cells in nests surrounded by a dense mononuclear infiltrate in the superficial dermis as well as hair follicles, (2) dermic pigment collections, (3) fibrotic replacement of hair follicles, and (4) positive Alcian blue staining in the superficial dermis and around some hair follicles.

One month after excision, follicle regrowth was noted. The same phenomenon was previously reported by Yesudian and Thambiah [2] and Beltrán and Bravo [7].

\section{Conclusion}

Perinevoid alopecia is an extremely rare clinical presentation of AA associated with a central, generally pigmented nevus where a cellular inflammatory response is triggered against hair follicles, nevoid cells, and melanocytic structures.

\section{Statement of Ethics}

This case report followed the Hospital ethics and investigation guidelines. The patient gave his informed consent to the publication of his case and the images taken during the medical treatment.

\section{Disclosure Statement}

The authors declare that they have no conflicts of interest to disclose.

\section{References}

1 Babu A, Bhat M, Dandeli S, Ali N: Throwing light onto the core of a halo nevus: a new finding. Indian J Dermatol 2016;61:238.

2 Yesudian P, Thambiah AS: Perinevoid alopecia. An unusual variety of alopecia areata. Arch Dermatol 1976;112:1432-1434.

3 Trautman S, Thompson M, Roberts J, Thompson C: Melanocytes: A possible autoimmune target in alopecia areata. J Am Acad Dermatol 2009;61:529-530.

4 Paus R, Slominski A, Czarnetzki BM: Is alopecia areata an autoimmune-response against melanogenesis-related proteins, exposed by abnormal MHC class I expression in the anagen hair bulb? Yale J Biol Med 1993; 66:541-554.

5 Gilhar A, Assy B, Shalaginov R, Serafimovich S, Landau M, Kalish R: Melanocyte-associated $\mathrm{T}$ cell epitopes can function as autoantigens for transfer of alopecia areata to human scalp explants on Prkdc(scid) mice. J Invest Dermatol 2001;117:1357-1362.

6 Mastruserio DN, Cobb MA, Ross VE: Nevocellular nevus associated with alopecia presenting as aplasia cutis congenita. Int J Dermatol 1998;37:37-38.

7 Beltrán GG, Bravo PF: Alopecia areata nevocéntrica. Dermatol Per 1998;8:47-49. 